\title{
A reliable framework for laparoscopic colorectal surgery based on well-structured and standardized surgical and post-operative protocols
}

loannis G Gkionis ( $\boldsymbol{D}$ ioannisgionis@hotmail.com )

Venizeleio General Hospital of Heraklion, Crete, Greece https://orcid.org/0000-0002-1639-1654

Mathaios E. Flamourakis

Venizeleio General Hospital of Heraklion, Crete, Greece

Zafeiro I. Karafoulidou

Venizeleio General Hospital of Heraklion, Crete, Greece

Michail I. Giakoumakis

Venizeleio General Hospital of Heraklion, Crete, Greece

Aggelos Laliotis

Venizeleio General Hospital of Heraklion, Crete, Greece

Eleni S. Tsagkataki

Venizeleio General Hospital of Heraklion, Crete, Greece

Eleni I. Kaloeidi

Venizeleio General Hospital of Heraklion, Crete, Greece

Andreas F. Strehle

Venizeleio General Hospital of Heraklion, Crete, Greece

Emmanouil N. Bachlitzanakis

Venizeleio General Hospital of Heraklion, Crete, Greece

Georgios P. Matzakanis

Venizeleio General Hospital of Heraklion, Crete, Greece

Athanasios K. Alegkakis

University of Crete Voutes Campus: Panepistemio Kretes Panepistemioupole Bouton

Manousos S. Christodoulakis

Venizeleio General Hospital of Heraklion, Crete, Greece

\section{Research Article}

Keywords: Colorectal surgery, laparoscopic surgery, standardization, learning curve, outcomes assessment

Posted Date: February 21st, 2022 
DOI: https://doi.org/10.21203/rs.3.rs-1291366/v1

License: (c) (1) This work is licensed under a Creative Commons Attribution 4.0 International License. Read Full License 


\section{Abstract}

Background: Although the number of colorectal operations performed laparoscopically has increased, it has not yet surpassed the number of laparotomies. The purpose of this single centre study was to determine, using various parameters and following well-structured and standardized surgical and postoperative procedures the safety and clinical outcomes of laparoscopic colorectal resections performed in a regional hospital by a team of surgeons supervised by a specialized in laparoscopic colorectal surgery. Through this process, it was given the opportunity to evaluate the impact of the learning curve by comparing their performance.

Methods: From 2012 to 2021, a total of 234 patients with pathology in the colon and rectum underwent laparoscopic colorectal resection. Patients' case records and histopathology reports were examined for predefined parameters, statistically analysed and compared.

Results: The type of operation had a significant effect on operative time $(p<0.001)$. When the duration of operation was correlated to the number of hospitalization days, a significant positive association was found $(r=0.173, p=0.012)$. The patient's age showed to affect operative time in a proportional way $(p=0.004)$, while the reverse is true for the number of days after $1^{\text {st }}$ surgery $(p<0.001)$. A reduction in operative times was observed for both the specialized and non-specialized surgeons $(r=-0.315, p<0.001$, $r s=-0.324, p<0.001)$, with a larger decline for specialized surgeon $(r=-0.322, p<0.001, r s=-0.360, p<0.001)$.

Conclusions: Taking into consideration all the parameters, it is our belief that through the framework we have implemented, any surgeon can complete uneventfully the learning curve of laparoscopic colorectal surgery, even in a regional hospital.

\section{Background}

Colorectal cancer (CRC) is an ever-increasing health concern, affecting millions of people worldwide with high rates of morbidity and mortality [1, 2]. Each year nearly 450,000 people are diagnosed with colorectal cancer in Europe, making CRC the most common cancer in the European Union [3]. At the same time, CRC is the third most commonly diagnosed malignancy and the second leading cause of cancer-related deaths worldwide [4]. It is expected that by $2030 \mathrm{CRC}$ incidence will increase to more than 2.2 million new cases per year, which would signify a 60\% rise, with 1.1 million cancer related deaths [2].

When preventive measures and early detection are either not implemented or have proved unsuccessful, and therefore CRC develops, surgical intervention emerges as the next step in the therapeutic cascade. Surgery remains the mainstay of treatment for most patients with CRC and is the principal constituent in every curative treatment in this cancer patient group $[5,6]$. Consequently, a great need for a minimally invasive curative surgical intervention, offering more favourable operative and post-operative outcomes, was never before deemed as important as it is today. 
Laparoscopic colectomy has emerged as a promising interventional means, offering equivalent oncological outcomes to conventional-open surgery and it has surpassed open surgery regarding shortterm outcomes [7-16]. The first laparoscopically assisted colectomy to be reported was in 1991 [17]. Three decades later, laparoscopic colectomy has proved invaluable in the treatment of CRC with a substantial number of clinical trials reporting on its significance [7-16]. These meta-analyses and randomised controlled studies demonstrated that laparoscopic colorectal surgery offers lesser postoperative pain, fewer cases of post-operative ileus with subsequent earlier return of bowel function, as well as fewer analgesic requirements and shorter hospital stay compared to open surgery [9-16].

In recent years, the number of colorectal operations performed laparoscopically has increased, but it has not yet surpassed the number of laparotomies. According to recent statistical data, the percentage of colorectal surgeries performed laparoscopically worldwide varies significantly, ranging from $20-50 \%[17$, 18]. This can be explained by the lengthy period required for a surgeon to reach the steep learning curve in laparoscopic colorectal surgery [19-21]. A learning curve is considered completed when the predefined variables reach a steady state and the surgical outcomes are comparable with those reported in the literature $[22,23]$.

The aim of this study was dual. Firstly, to determine the safety and clinical outcomes of laparoscopic resections performed in a regional hospital, where a well-structured and standardized surgical technique and postoperative protocols are being followed; thus, providing a reliable framework which any surgeon who undertakes laparoscopic colectomy should follow. Secondly, to analyse the impact of the learning curve in laparoscopic colorectal surgery by comparing the surgical outcomes in operations performed by a surgeon who has reached the learning curve, and, therefore, is considered specialized, with those operations supervised by him. The latter were performed by surgeons who were at the beginning of their learning curve in laparoscopic colorectal surgery.

\section{Methods}

From October 2012 to April 2021, 234 patients with pathology in the rectum and colon underwent laparoscopic resection at a regional general hospital in Greece (Venizeleio General Hospital of Heraklion in Crete). The operations were performed by a number of surgeons who were all part of a team attended and supervised by an experienced surgeon in laparoscopic colorectal surgery (M.C.).

Patients with a locally advanced disease (T4 or bulky tumours) or with previous open operations with a midline incision, and those with a BMI over 35 were excluded from the study. The case records and histopathology reports were scrutinised for predefined parameters such as patient demographic data, the location of tumour, the type of surgical procedure, conversion to open surgery, the surgical time, the distal and circumferential margins, the number of harvested lymph nodes, and the overall length of hospital stay. Mortality, any surgical complications, as well as oncological outcomes were also examined. The data collected (additional file) were subsequently statistically analysed. 
The surgical time was calculated from the moment of the first laparoscopic port placement until wound closure. The data for all the operations were collected from the formal surgery records. Operations which were converted to open surgery were excluded from the surgical time analysis. The distal and circumferential margins, and the number of harvested lymph nodes were obtained from the histopathology reports. Circumferential resection margins (CRM) were defined as positive when malignant cells were found microscopically at a distance of less than $2 \mathrm{~mm}$.

\section{Preoperative preparation}

All patients included in this study underwent a preoperative preparation which included the following: an extended medical report, a thorough physical examination including a digital rectal examination, blood tests for identifying cancel marker levels, a chest $\mathrm{X}$-ray, thoracic and abdominal computerized tomography, as well as complete colonoscopy with biopsies taken from the lesions. Patients with rectal tumours underwent rigid rectoscopy along with nuclear magnetic resonance imaging (MRI) of the rectum. When preoperative chemoradiotherapy was conducted in a subgroup of patients with rectal cancer, a repetitive MRI was performed. Additionally, all patients underwent bowel preparation preoperatively and were administered a single intravenous injection of a double antibiotic combination prior to surgery, followed by the intravenous administration of the same antibiotic combination, three times daily for two days postoperatively.

\section{Surgical notes}

The surgical procedures were performed by a team of surgeons supervised by a specialized surgeon (M.C.) who has completed the learning curve in laparoscopic colorectal surgery [24]. A well-structured and standardized surgical procedure was implemented in all instances, strictly following oncological criteria, according to the principles of complete mesocolic excision (CME) and total mesorectal excision (TME) with central vascular ligation (CVL) [25-27]. Furthermore, the splenic flexure was mobilised to all patients who underwent low anterior resections and left colectomies in order to limit tension on the colorectal anastomosis. For colorectal anastomoses with a distance of less than $4 \mathrm{~cm}$ from the anal verge, a diverting loop ileostomy was created. The same surgical protocol was followed both for the colectomies performed by the specialized surgeon and the operations executed by any other member of the team with the attendance of the specialized surgeon.

General anaesthesia with concurrent epidural analgesia was the preferred mode of surgical anaesthesia. In the subgroup of patients with a tumour located in the lower rectum, which was unsuitable for low anterior resection, extralevator abdominoperineal resection (ELAPE) was the chosen surgical technique, with the patient placed in a jack knife position [28, 29].

\section{Postoperative protocol}

All patients in this study were submitted to a "fast track" postoperative protocol. Specifically, early patient mobilization was encouraged with the patients being ambulatory the morning following the day of surgery. None of the patients had a nasogastric tube inserted either pre- or postoperatively, therefore, all 
of them were able to start drinking liquids from the first postoperative day. The urinary catheter was removed the morning after the operation, with the exception being patients who underwent ELAPE or low anterior resection. In those cases, the catheter was kept in place for at least four additional days.

\section{Statistical Methods}

Mean, median and standard deviation (SD) were used to describe the continuous variables such as operation hours and days of hospitalization, while frequencies and \%frequencies were used for discrete data. Independent samples t-test and the corresponding non-parametric Mann-Whitney were used for comparison between two groups. When it was necessary to compare more than two groups, one-way ANOVA was used. Pearson chi-square test was assessed to examine possible association between two discrete variables. Box plots and scatter plots were used for graphical representation of the data. Multiple linear regression was applied to establish predictors of operation time versus explanatory variables. IBM SPSS Statistics 26.0 was used for statistical analysis of the data and $a=0.05$ was set as the minimum acceptance level of significance.

\section{Results}

A total of 234 patients underwent laparoscopic colorectal surgery by a team of surgeons at a public regional hospital (Venizeleio General Hospital of Heraklion, Crete, Greece) in a time period spanning from October 2012 to April 2021. From these 234 patients who were initially included in the study, 22 (9\%) were excluded due to conversion from laparoscopic to open surgery.

The distribution of surgeries showed that $81(38.2 \%)$ were right colectomies, followed by 68 sigmoidectomies (32.1\%), 26 low anterior resections (12.3\%), 18 ELAPE (8.5\%), 15 left colectomies (7.1\%) and, finally, 4 subtotal colectomies (1.9\%) (Figure 1).

Most patients were males (54.2\%), with a mean age of $65.9 \pm 12.9$ years, and 126 of them $(59.4 \%)$ belonged in the age group of $60-79$. In only 3 operations (1.4\%), oncological margins were infiltrated, while complications were present in 13 cases (6\%). The majority of operations were performed by the specialized surgeon, 151 in number, accounting for $71.2 \%$ of total cases, while in the rest of them the specialized surgeon supervised the non-specialized members of the team of surgeons (Table 1). 
Table 1

Demographic and clinical characteristics

\begin{tabular}{|c|c|c|c|}
\hline & & $\mathrm{n}$ & $\%$ \\
\hline \multirow[t]{2}{*}{ Sex } & Man & 115 & $54.2 \%$ \\
\hline & Woman & 97 & $45.8 \%$ \\
\hline \multirow[t]{5}{*}{ Age Groups } & $<50$ & 24 & $11.3 \%$ \\
\hline & $50-59$ & 32 & $15.1 \%$ \\
\hline & $60-69$ & 63 & $29.7 \%$ \\
\hline & $70-79$ & 63 & $29.7 \%$ \\
\hline & $80+$ & 30 & $14.2 \%$ \\
\hline \multicolumn{2}{|c|}{ Age (mean \pm SD) (range) } & $65.9 \pm 12.2$ & $(33-88)$ \\
\hline \multirow[t]{2}{*}{ Surgeon } & Specialized & 151 & $71.2 \%$ \\
\hline & Non-Specialized & 61 & $28.8 \%$ \\
\hline \multirow[t]{2}{*}{ Margins } & Infiltrated margins & 3 & $1.4 \%$ \\
\hline & Free margins & 209 & $98.6 \%$ \\
\hline \multirow[t]{2}{*}{ Complications } & Yes & 13 & $6,1 \%$ \\
\hline & No & 199 & $93,9 \%$ \\
\hline
\end{tabular}

The distribution of different types of colectomies per year is depicted in figure 2, whereas figure 3 shows the number of cases performed each year in conjunction with the surgical specimen. In the first 4 years the number of right colectomies was higher than any other type of surgical procedure, followed by sigmoidectomies. This pattern has changed in the last 6 years, as the number of right colectomies and sigmoidectomies is the same, with increasing cases of low anterior resections and left colectomies. Additionally, in most of the cases performed throughout the years, the pathological examination of the surgical specimen revealed a tumour stage $\leq \mathrm{T} 3$. Colectomies regarding tumours stage $>\mathrm{T} 3$ were performed for the first time during the fourth year.

In Table 2 the effect of the type of operation on operative time proved to be significant $(F(5,206)=13.378$, $\mathrm{p}<0.001$ ). Subtotal colectomies showed the highest mean operation time $5.9 \pm 1.3 \mathrm{~h}$ (median $6.3 \mathrm{~h}$ ), followed by ELAPE surgeries (mean $5.1 \pm 1.2 \mathrm{~h}$, median $5.5 \mathrm{~h}$ ). Left colectomies lasted $2.5-4.5 \mathrm{~h}$ with a mean of $3.3 \pm 0.6 h$. 
Table 2

Differences in operative times versus type of operation

\begin{tabular}{|lllll|}
\hline Type of Operation & $\mathbf{N}$ & Mean & Median & Min-Max \\
\hline ELAPE & 18 & $5.1 \pm 1.2$ & 5.5 & $2.0-.7 .0$ \\
\hline Sigmoidectomy & 68 & $3.5 \pm 1.0$ & 3.5 & $2.0-8.0$ \\
\hline Right colectomy & 81 & $3.5 \pm 1.2$ & 3.5 & $2.0-10.0$ \\
\hline Low interior resection & 26 & $4.7 \pm 1.3$ & 4.5 & $3.5-10.0$ \\
\hline Left colectomy & 15 & $3.3 \pm 0.6$ & 3.5 & $2.5-4.5$ \\
\hline Subtotal colectomy & 4 & $5.9 \pm 1.3$ & 6.3 & $4.0-7.0$ \\
\hline Total & 212 & $3.8 \pm 1.3$ & 3.5 & $2.0-10.0$ \\
\hline & $F(5,206)=13.378$. & $p<0.001$ & \\
\hline
\end{tabular}

Tables 3 and 4 represent the surgical complications for each type of operation performed by the specialized and non-specialized surgeons. In each of these 13 cases, a laparotomy was performed following the initial laparoscopic colorectal operation. Additionally, a single patient who exhibited anastomotic leakage following laparoscopic sigmoidectomy passed away during the postoperative period after the second operation, due to deterioration of the respiratory system. No other deaths were reported during the laparoscopic operations or the immediate postoperative period.

Table 3

Description of complications between specialized and nonspecialized surgeons

\begin{tabular}{|lll|}
\hline Complications & Specialized & Non-specialized \\
\hline Anastomotic leakage & 5 & 2 \\
\hline Anastomotic stenosis & 2 & 1 \\
\hline Ligature of the ureter & 1 & 0 \\
\hline Lesion of the urinary bladder & 1 & 0 \\
\hline Lesion of the iliac vein & 1 & 0 \\
\hline TOTAL NUMBER & 10 & 3 \\
\hline
\end{tabular}


Table 4

Description of complications for each type of surgery

\section{Complications Type of Surgery}

\begin{tabular}{|lllllll|} 
& $\begin{array}{l}\text { Right } \\
\text { colectomy }\end{array}$ & Sigmoidectomy & $\begin{array}{l}\text { Low } \\
\text { anterior } \\
\text { resection }\end{array}$ & $\begin{array}{l}\text { ELAPE } \\
\text { Left } \\
\text { colectomy }\end{array}$ & $\begin{array}{l}\text { Subtotal } \\
\text { colectomy }\end{array}$ \\
$\begin{array}{l}\text { Anastomotic } \\
\text { leakage }\end{array}$ & - & 3 & 2 & - & 1 & 1 \\
$\begin{array}{l}\text { Anastomotic } \\
\text { stenosis }\end{array}$ & - & 1 & 1 & - & 1 & - \\
$\begin{array}{l}\text { Ligature of the } \\
\text { ureter }\end{array}$ & 1 & - & - & - & - & - \\
$\begin{array}{l}\text { Lesion of the } \\
\text { urinary bladder }\end{array}$ & - & 1 & - & - & - & - \\
\hline $\begin{array}{l}\text { Lesion of the iliac } \\
\text { vein }\end{array}$ & - & 1 & - & - & - & - \\
\hline \begin{tabular}{l} 
TOTAL NUMBER \\
\hline
\end{tabular} & 1 & 6 & 3 & 0 & 2 & 1 \\
\hline
\end{tabular}

A low but significant positive association was found when the duration of operation was correlated to the number of hospitalisation days $(r=0.173, p=0.012, r s=0.250, p<0.001)$ and patient's age, but it was not correlated with the number of harvested lymph nodes $(r=-0.059, p=0.395$. $r s=-0.114, p=0.099)$ (Figure 4, ac).

Table 5 shows the results of multiple linear regression on operative time from a set of explanatory variables. The patient's age showed to affect operating time $(b=0.02,0.01-0.03, p=0.004)$, while a reduction was found for the number of days after 1 st surgery $\left(b=-4.010^{4}, 5.610^{-4}--2.410^{-4}, p<0.001\right)$. ELAPE showed a higher mean operating time in comparison to sigmoidectomy $(b=1.31,0.78-1.85$, $p<0.001)$, while the same was true for low anterior resections $(b=1.13,0.70-1.55)$ and subtotal colectomies $(b=2.49,1.44-3,54, p<0.001)$. 
Table 5

Predictors of operating time

\begin{tabular}{|llllll|}
\hline & \multicolumn{2}{l}{$\begin{array}{l}\text { Unstandardized } \\
\text { Coefficients }\end{array}$} & \multicolumn{3}{l|}{$\begin{array}{l}\text { Standardized } \\
\text { Coefficients }\end{array}$} \\
\cline { 2 - 6 } & B & $95 \%$ LB & $95 \%$ UB & Beta \\
\hline $\begin{array}{l}\text { Surgeon (Specialized or non } \\
\text { specialized) }\end{array}$ & 0.03 & -0.03 & 0.09 & 0.049 & 0.370 \\
\hline Patient's age (years) & 0.02 & 0.01 & 0.03 & 0.157 & 0.004 \\
\hline Days from 1st surgery & $-4.0 \mathrm{E}-$ & $-5.6 \mathrm{E}-$ & $-2.4 \mathrm{E}-04$ & -0.279 & $<0.001$ \\
\hline Operation=ELAPE & 04 & 04 & & & $<0.001$ \\
\hline Operation=Right colectomy & 1.31 & 0.78 & 1.85 & 0.284 & 0.320 \\
\hline Operation=Low interior resection & -0.16 & -0.49 & 0.16 & -0.063 & $<0.001$ \\
\hline Operation=Left colectomy & 1.13 & 0.70 & 1.55 & 0.306 & 0.696 \\
\hline Operation=Subtotal colectomy & 0.11 & -0.44 & 0.66 & 0.023 & $<0.001$ \\
\hline
\end{tabular}

Table 6 summarizes the age of patients, parameters of surgery (complications, infiltrated margins, operation duration and harvested lymph nodes) and days of hospitalisation in operations performed either by the specialized or the non-specialized surgeons. Except for the mean operation duration, which is lengthier in operations performed by the specialized surgeon $(3.9 \pm 1.4)$ in comparison to the nonspecialized (3.5 \pm 1.0$)$, no other surgical parameter showed a level of significance less than 0.05 . When the surgeon (specialized/non-specialized) was considered in relation to the type of operation in a two way ANOVA (surgeon, type of operation), no significant results were found for Operation $x$ Surgeon $(F(5$, 200) $=0,918, p=0,470$, Surgeon $F(1,200)=1,164, p=0,558)$. 
Table 6

Differences in surgical parameters and age of patients between specialized and nonspecialized surgeons

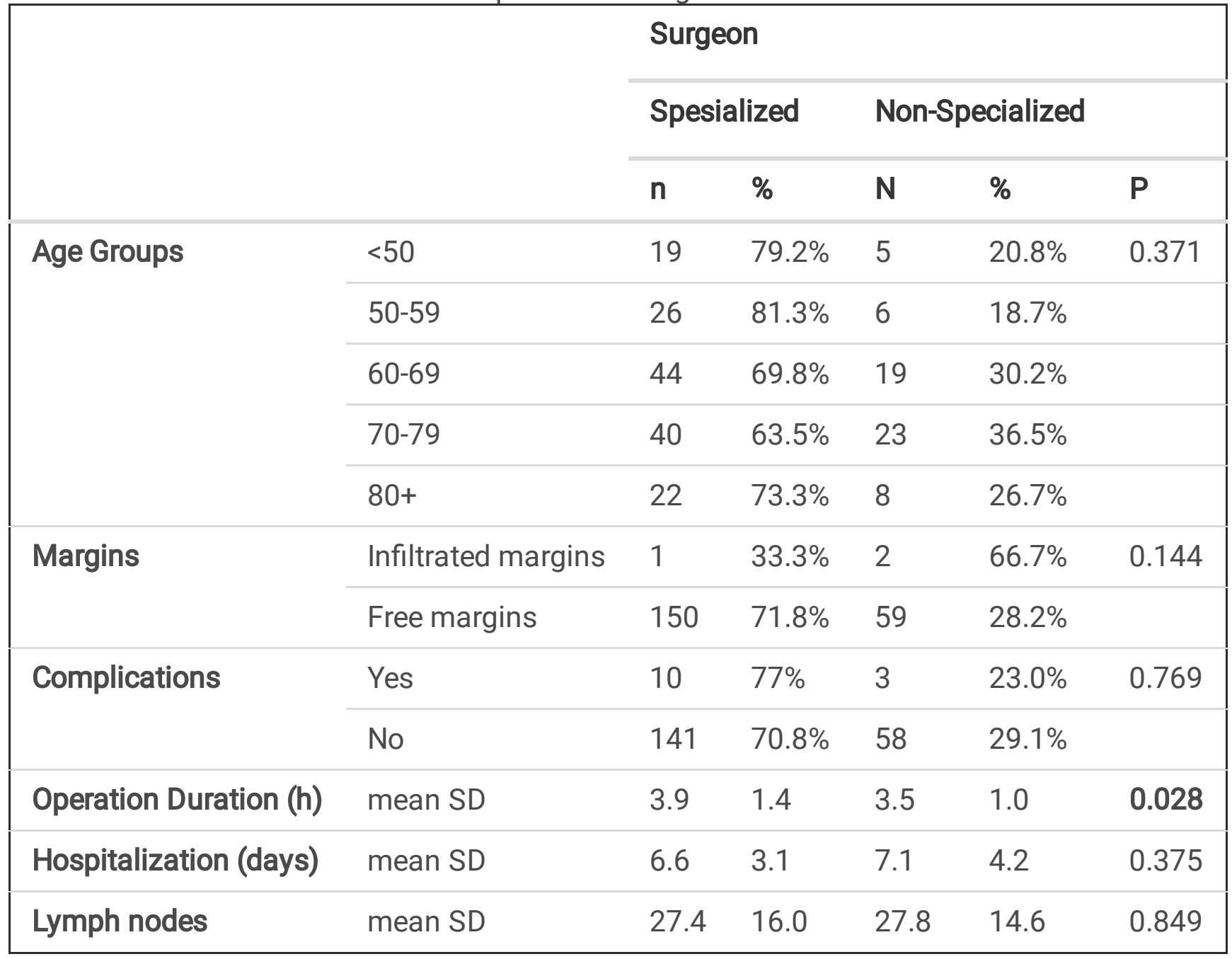

Figure 5 depicts the learning effect expressed as time (in days) of the operation date minus the $1 \mathrm{st}$ recorded operation date. A low to medium negative association of operating time and days after $1 \mathrm{st}$ surgery was found $(r=-0.315, p<0.001, r s=-0.324, p<0.001)$. When applied separately to the cases performed by the specialized and non-specialized surgeons, the same negative association was present in the operations performed by the specialized surgeon ( $r=-0.322, p<0.001, r s=-0.360, p<0.001)$, but not on those performed by the non-specialized surgeons $(r=-0.205, p=0.113, r s=-0.171, p=0.189)$.

\section{Discussion}

Four large prospective, randomized controlled trials, from North America, Canada and Europe have demonstrated that laparoscopic treatment of colorectal cancer yields oncologic results similar to those of open surgery, with no increased morbidity or mortality [10-15]. Furthermore, the laparoscopic approach offers lesser post-operative pain, fewer cases of post-operative ileus, shorter hospital stay and reduced hospital costs [7-16]. 
In the present study, resection margins were infiltrated in only 3 cases, accounting for $1.4 \%$ of total cases, while intra-operative complications occurred in 13 of them $(6,1 \%)$. From the total 234 laparoscopic colorectal operations performed during the time period 2012-2021, 22 were converted to open intraoperatively, accounting for $9 \%$ of the total. Our overall $9 \%$ conversion rate is in accordance with the 5$20 \%$ reported in the literature [30-32]. Specifically, a multicentre study performed by Rose et al. reported an occurrence of intra-operative complications in $5.9 \%$ of laparoscopic colorectal operations, whereas a conversion to open surgery occurred in $5.6 \%$ of cases [32]. In addition, the $0.4 \%$ overall mortality rate and the $3,3 \%$ anastomotic leak rate for laparoscopic surgeries, which were detected in our study, are comparable with those in multicentre trials [30-32].

Based on the results of our study, greater intraoperative time can lead to an increase in the number of hospitalization days post-operatively, without having an impact on the number of harvested lymph nodes. A study performed by Harrison et al. supports this evidence, correlating operative time and number of hospitalisation days in a proportional way, whilst Scheer et al. found no significant association between the aforementioned parameters [33,34]. In addition, the present study showed that the specific type of laparoscopic colorectal operation performed had an effect on operative time; with subtotal colectomies having the highest mean operative time, left colectomies the lowest, and ELAPE falling somewhere in between.

In our study the patient's age, the number of performed operations and the surgeon's expertise proved to affect intra-operative time, regardless of the type of operation. As the patient's age increased, the duration of operation increased as well. A study performed by Lim et al. demonstrated that the patient's age affects operative time in a reversible proportional way, reporting a significant reduction in the elderly surgical patients [35]. Conversely, Ueda et al. did not identify considerable differences in operative time based solely on the patient's age [36].

The operation time in laparoscopic colorectal surgery is longer than that in open surgery even if the resections are performed by surgeons with experience in advanced laparoscopic procedures [37]. In this study, a reduction in operative times was observed for both the specialized and non-specialized surgeons, being proportional to the number of operations performed by each of them. The operative time decreases with the surgeon's increasing experience, and it is a useful criterion for evaluating the learning curve [24]. At the same time, the specialized surgeon proved to have a greater reduction in operative times compared to the non-specialized surgeons. It has been previously showed that the surgeon's expertise is reversible proportional to the operative time indicating the point where the learning curve is completed by reaching a steady level of performance $[24,38,39]$.

Apart from the duration of operation, no other difference was observed between the specialized and nonspecialized surgeons. A possible explanation is the implementation of well-structured and standardized surgical technique and postoperative protocol which were carefully followed by all members of the surgical team. Additionally, the assistance of the specialized surgeon may act as safety valve for the adequacy and the oncological safety of the laparoscopic colectomies performed by surgeons who are at 
the beginning of their learning curve. Similar conclusions were reached by Luglio et al. and Akiyoshi et al. with both studies reporting no negative impact on short-term outcomes when the operations were performed by trainees under the supervision of a highly experienced surgeon in laparoscopic colorectal surgery $[27,40]$.

Furthermore, it is advisable for surgeons who are in the beginning of their learning curve to be selective in their first cases $[27,40]$. Right colectomies and sigmoidectomies regarding tumours stage $\leq \mathrm{T} 3$ are the preferable operations in the beginning; more technically advanced and demanding resections, such as low anterior resections, left colectomies, subtotal colectomies, and operations for tumours stage $>\mathrm{T} 3$, is advisable to be performed later in a surgeon's laparoscopic colorectal journey, when they will have gained the necessary technical skills and will have adapted to the new laparoscopic setting. Moreover, performing a smaller number of operations in the beginning, whilst increasing in quantity and difficulty every year, may facilitate the completion of the learning curve, as the surgeon will have the time to process and adopt the new skills and knowledge, respectively. Last but not least, the ample knowledge of anatomy and the respect for the rules of surgical oncology are two required prerequisites for successful occupation with laparoscopic colorectal surgery.

\section{Conclusions}

Laparoscopic colorectal surgery is a safe and minimally invasive procedure for the surgical treatment of pathological entities of the colon and rectum, offering significant advantages in comparison to open surgery in terms of short-term outcomes. Specific patient and surgeon characteristics, as well as surgical parameters, can influence clinical outcomes, intra-operative time, and oncologic efficacy. The patient's age, the number of performed operations and the surgeon's expertise affect intra-operative time, which in turn has an impact on the number of hospitalization days post-operatively. A surgeon's expertise influence the duration of operation, but does not compromise short-term outcomes, if the presence of a specialized supervisor is guaranteed intraoperatively. Therefore, the patient's safety and adequacy of surgical resection are not jeopardised. Based on the results of this study, we advocate the use of laparoscopic colorectal surgery in the treatment of colorectal cancer even by non-specialized surgeons when a framework based on well-structured and standardized surgical and post-operative protocols, just like the one we have presented, is implemented.

\section{Abbreviations}

$\cdot \mathrm{CRC}$

- Colorectal cancer

- M.C.

- Manousos Christodoulakis

- CRM

- Circumferential resection margins

-MRI 
- Magnetic resonance imaging

- CME

- Complete mesocolic excision

- TME

- Total mesorectal excision

- CVL

- Central vascular ligation

- ELAPE

- Extralevator abdominoperineal resection

-SD

- Standard deviation

\section{Declarations}

Ethics approval and consent to participate: This study was conducted in accordance with the ethical standards of the institutional and national research committee and with the 1964 Helsinki Declaration and its later amendments or comparable ethical standards. The study was approved by the Ethics Committee of the Scientific Board of Venizeleio General Hospital of Heraklion (Decision No. 126-subject 1/ Meeting 20/26-10-2021). Informed consent was obtained from all patients. The consent was either written or verbal. From some patients, verbal consent was obtained due to either a low level of educational attainment or a better understanding of the scientific protocol of the study using oral language. In these cases, a member of the Ethics Committee of the Scientific Board of Venizeleio General Hospital of Heraklion was always present, in order to ensure the understanding of the scientific protocol of the study from the patients and to certify that they give their consent. The Ethics Committee of the Scientific Board of Venizeleio General Hospital of Heraklion, with the same decision (Decision No. 126subject 1/ Meeting 20/26-10-2021) accepted both methods (written or verbal) for providing information to and obtaining consent from the patients.

Consent for publication: Not applicable.

Availability of data and material: The datasets generated and analysed during the current study are included in this manuscript and its additional files.

Competing interests: The authors declare no conflicts of interest.

Funding: There was no funding.

Authors' contributions: All authors contributed to the study conception and design. Data collection and analysis were performed by IG, MF, MG, ET, EK and AA. MC supervised the project. The first draft of the manuscript was written by IG in consultation with $\mathrm{ZK}, \mathrm{AL}, \mathrm{AS}, \mathrm{EB}$ and $\mathrm{AA}$. All authors commented on previous versions of the manuscript. All authors read and approved the final manuscript. 
Acknowledgements: Not applicable.

\section{References}

1. SiegelRL, Miller KD, Sauer AG et al. (2020) Colorectal cancer statistics, 2020. Cancer J Clin. 70(3):145-164

2. Arnold M, Sierra MS, Laversanne M et al. (2017) Global patterns and trends in colorectal cancer incidence and mortality. Gut. 66(4):683-691

3. Høydahl O, EdnaTH, Xanthoulis A et al. (2020) Long-term trends in colorectal cancer: incidence, localization, and presentation. BMC Cancer 20(1):1077

4. Loomans-Kropp HA, UmarA (2019) Increasing Incidence of Colorectal Cancer in Young Adults. J Cancer Epidemiol. 2019:9841295

5. Safiri S, Sepanlou SG, Ikuta KS et al. (2019) The global, regional, and national burden of colorectal cancer and its attributable risk factors in 195 countries and territories, 1990-2017: a systematic analysis for the Global Burden of Disease Study 2017. Lancet Gastroenterol Hepatol. 4(12):913-933

6. Cardoso R, Guo F, Heisser T et al. (2021) Colorectal cancer incidence, mortality, and stage distribution in European countries in the colorectal cancer screening era: an international population-based study. Lancet Oncol. 22(7):1002-1013

7. Bonnor RM, Ludwig KA (2005) Laparoscopic Colectomy for Colon Cancer: Comparable to Conventional Oncologic Surgery? Clin Colon Rectal Surg. 18(3):174-81

8. Nelson H, Sargent DJ, Wieand HS et al. (2004) A comparison of laparoscopically assisted and open colectomy for colon cancer. N Engl J Med. 350(20):2050-9

9. Bonjer HJ, WCJ Hop, Nelson H et al. (2007) Laparoscopically assisted vs open colectomy for colon cancer: a meta-analysis. Arch Surg. 142(3):298-303

10. Van der Pas MH, Haglind E, Cuesta MA et al. (2013) Laparoscopic versus open surgery for rectal cancer (COLOR II): short-term outcomes of a randomised, phase 3 trial. Lancet Oncol. 14(3):210-8

11. Guillou PJ, Quirke P, Thorpe H et al. (2005) Short-term endpoints of conventional versus laparoscopicassisted surgery in patients with colorectal cancer (MRC CLASICC trial): multicentre, randomised controlled trial. 365(9472):1718-26

12. Veldkamp R, Kuhry E, Hop WCJ et al. (2005) Laparoscopic surgery versus open surgery for colon cancer: short-term outcomes of a randomised trial. Lancet Oncol. 6(7):477-84

13. Luglio G, Nelson H (2010) Laparoscopy for colon cancer: state of the art. Surg Oncol Clin N Am. 19(4):777-91

14. Fleshman J, Sargent DJ, Green E et al. (2007) Laparoscopic colectomy for cancer is not inferior to open surgery based on 5-year data from the COST Study Group trial. Ann Surg. 246(4):655-62 
15. Lacy AM, Garcia-Valdecasas JC, Delgado S et al. (2002) Laparoscopic assisted colectomy vs. open colectomy for treatment of non-metastatic colon cancer: a randomized trial. Lancet. 359(9325):22249

16. Hasegawa H, Kabeshima Y, Watanabe M et al. (2003) Randomised controlled trial of laparoscopic versus open colectomy for advanced colorectal cancer. Surg Endosc. 17(4):636-40

17. Laudicella M, Walsh B, Munasinghe A et al. (2016) Impact of laparoscopic versus open surgery on hospital costs for colon cancer: a population-based retrospective cohort study. BMJ Open. 6(11):e012977

18. Rea JD, Cone MM, Diggs BS et al. (2011) Utilization of laparoscopic colectomy in the United States before and after the clinical outcomes of surgical therapy study group trial. Ann Surg. 254(2):281-8

19. Schlachta CM, Mamazza J, Seshadri PA et al. (2001) Defining a learning curve for laparoscopic colorectal resections. Dis Colon Rectum. 44(2):217-222

20. Tekkis PP, Senagore AJ, Delaney CP et al. (2005) Evaluation of the learning curve in laparoscopic colorectal surgery: comparison of right-sided and left-sided resections. Ann Surg. 242(1):83-91

21. Park IJ, Choi GS, Lim KH et al. (2009) Multidimensional analysis of the learning curve for laparoscopic colorectal surgery: lessons from 1,000 cases of laparoscopic colorectal surgery. Surg Endosc. 23(4):839-846

22. Khan N, Abboudi H, Khan MS et al. (2014) Measuring the surgical 'learning curve': methods, variables and competency. BJU Int. 113(3):504-8

23. Pitiakoudis M, Michailidis L, Zezos $P$ et al. (2011) Quality training in laparoscopic colorectal surgery: does it improve clinical outcome? Tech Coloproctol. 15 Suppl 1:S17-20

24. Gkionis IG, Flamourakis ME, Tsagkataki ES et al. (2020) Multidimensional analysis of the learning curve for laparoscopic colorectal surgery in a regional hospital: the implementation of a standardised surgical procedure counterbalances the lack of experience. BMC Surg. 20(1):308

25. West NP, Hohenberger W, Weber K et al. (2010) Complete mesocolic excision with central vascular ligation produces an oncologically superior specimen compared with standard surgery for carcinoma of the colon. J Clin Oncol. 28(2):272-278

26. West NP, Sutton KM, Ingeholm P et al. (2010) Improving the quality of colon cancer surgery through a surgical education program. Dis Colon Rectum. 53(12):1594-603

27. Luglio G, De Palma GD, Tarquini R et al. (2015) Laparoscopic colorectal surgery in learning curve: Role of implementation of a standardized technique and recovery protocol. A cohort study. Ann Med Surg (Lond). 4(2):89-94

28. Kamali D, Sharpe A, Musbahi A et al. (2017) Oncological and quality of life outcomes following extralevator versus standard abdominoperineal excision for rectal cancer. Ann R Coll of Surg Engl. 99(5):402-409

29. Zhang Y, Wang D, Zhu L et al. (2017) Standard versus extralevator abdominoperineal excision and oncologic outcomes for patients with distal rectal cancer: A meta-analysis. Medicine (Baltimore). 96(52):e9150 
30. De Mulder W, Gillardin JP, Hofman P et al. (2001) Laparoscopic colorectal surgery. Analysis of the first 237 cases. Acta Chir Belg. 101(1):25-30

31. Köckerling F, Schneider C, Reymond MA et al. (1998) Early results of a prospective multicenter study on 500 consecutive cases of laparoscopic colorectal surgery. Laparoscopic Colorectal Surgery Study Group (LCSSG). Surg Endosc. 12(1):37-41

32. Rose J, Schneider C, Yildirim C et al. (2004) Complications in laparoscopic colorectal surgery: results of a multicentre trial. Tech Coloproctol. 8 Suppl 1:s25-8

33. Harrison OJ, Smart NJ, White P et al. (2014) Operative time and outcome of enhanced recovery after surgery after laparoscopic colorectal surgery. JSLS. 18(2):265-72

34. Scheer A, Martel G, Moloo H et al. (2009) Laparoscopic colon surgery: does operative time matter? Dis Colon Rectum. 52(10):1746-1752

35. Lim SW, Kim YJ, Kim HR (2017) Laparoscopic surgery for colorectal cancer in patients over 80 years of age: the morbidity outcomes. Ann Surg Treat Res. 92(6):423-428

36. Ueda Y, Shiraishi N, Kawasaki T et al. (2020) Short- and long-term outcomes of laparoscopic surgery for colorectal cancer in the elderly aged over 80 years old versus non-elderly: a retrospective cohort study. BMC Geriatr. 20(1):445

37. Lezoche E, Feliciotti F, Paganini AM et al. (2000) Laparoscopic colonic resections versus open surgery: a prospective non-randomized study on 310 unselected cases. Hepatogastroenterology. 47(33):697-708

38. Tsai KY, Kiu KT, Huang MT et al. (2016) The learning curve for laparoscopic colectomy in colorectal cancer at a new regional hospital. Asian J Surg. 39(1):34-40

39. Choi DH, Jeong WK, Lim SW et al. (2009) Learning curves for laparoscopic sigmoidectomy used to manage curable sigmoid colon cancer: single-institute, three-surgeon experience. Surg Endosc. 23(3):622-628

40. Akiyoshi T, Kuroyanagi $H$, Ueno $M$ et al. (2011) Learning curve for standardized laparoscopic surgery for colorectal cancer under supervision: a single-center experience. Surg Endosc. 25(5):1409-14

\section{Figures}



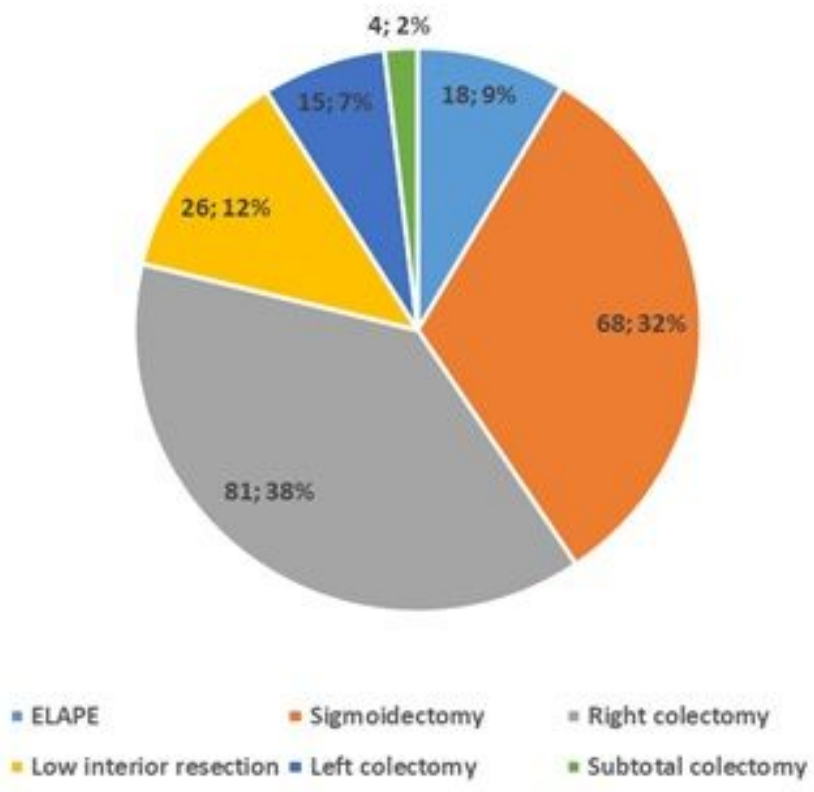

\section{Figure 1}

Distribution of different types of laparoscopic colorectal surgery

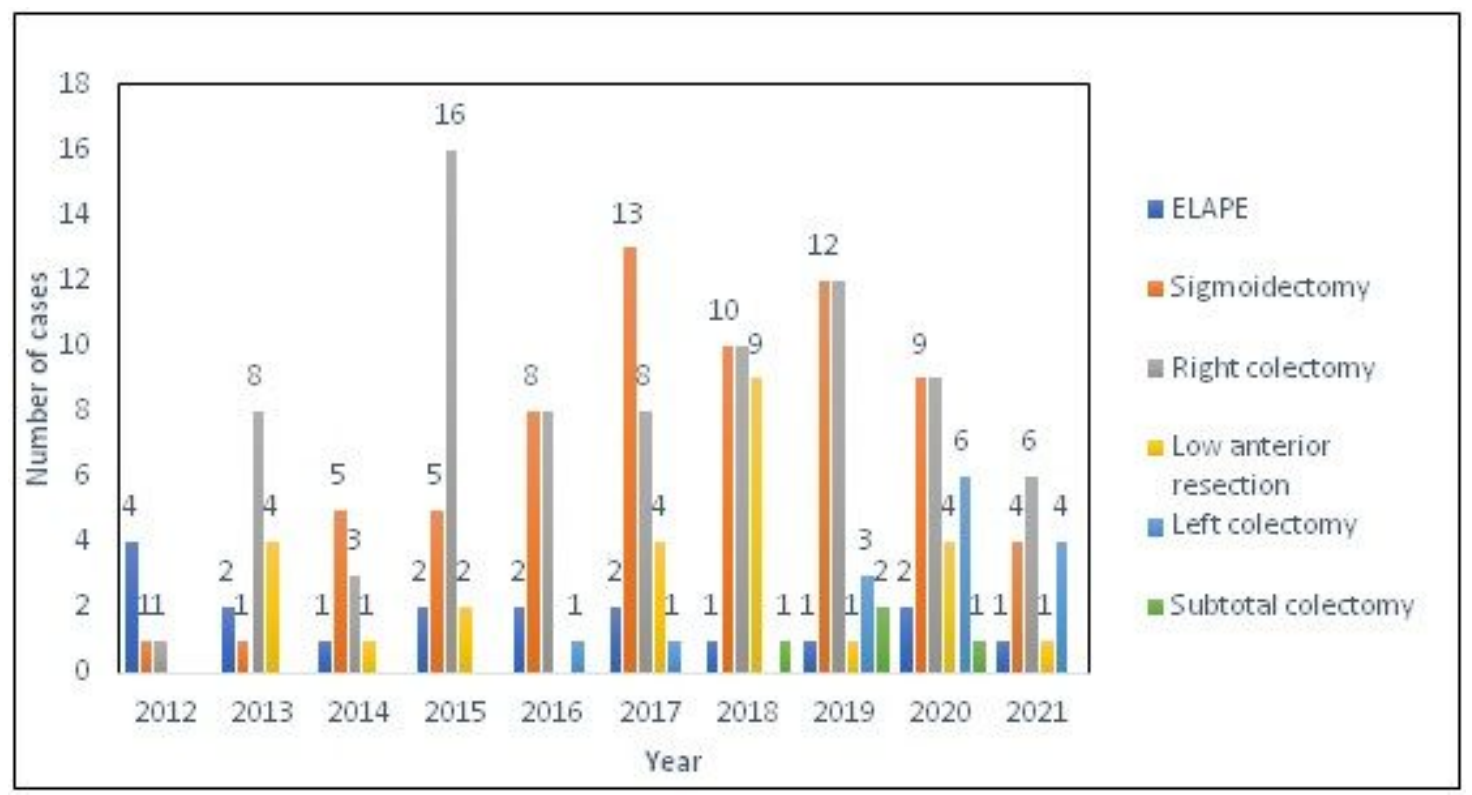

Figure 2

Distribution of different types of colectomies per year 


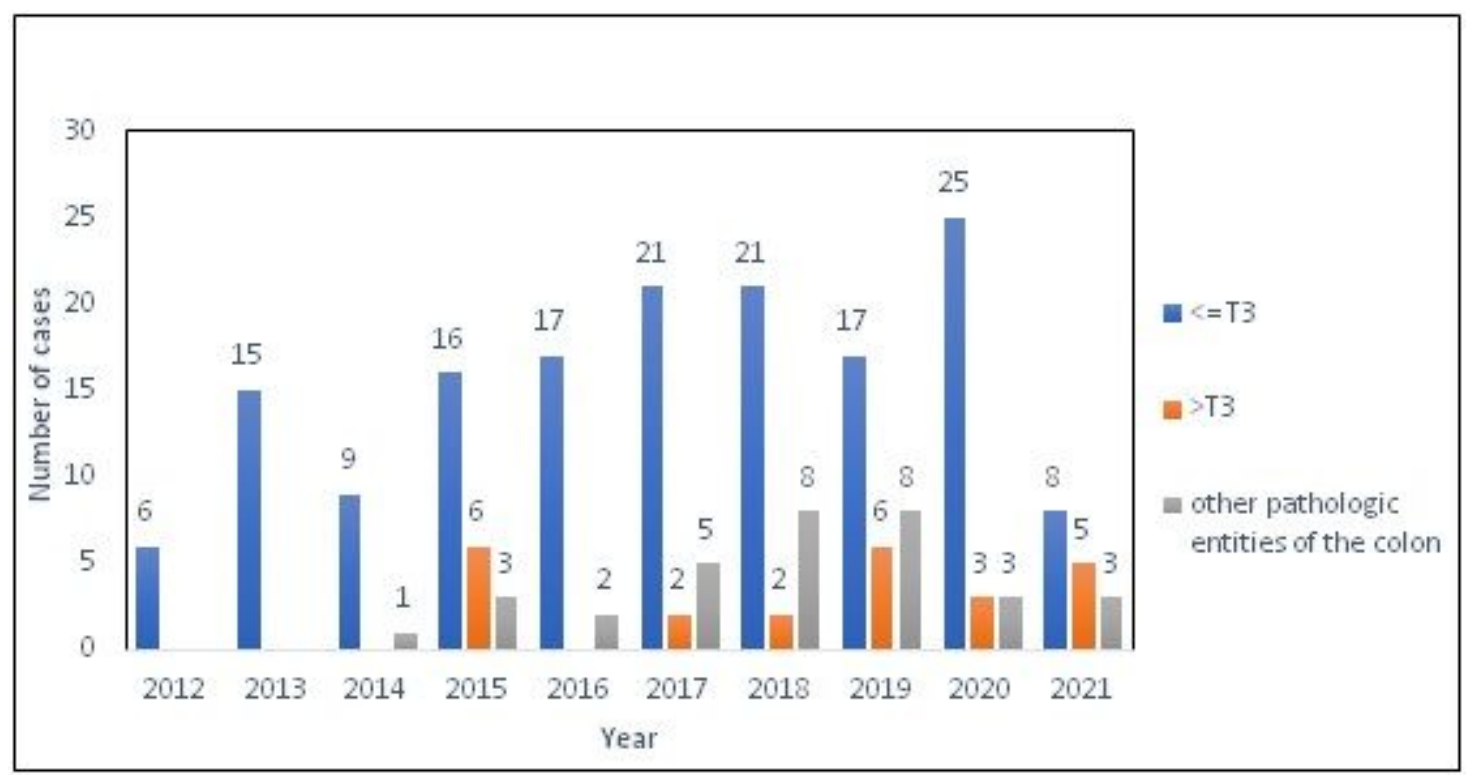

Figure 3

Number of cases per year in conjunction with the surgical specimen 

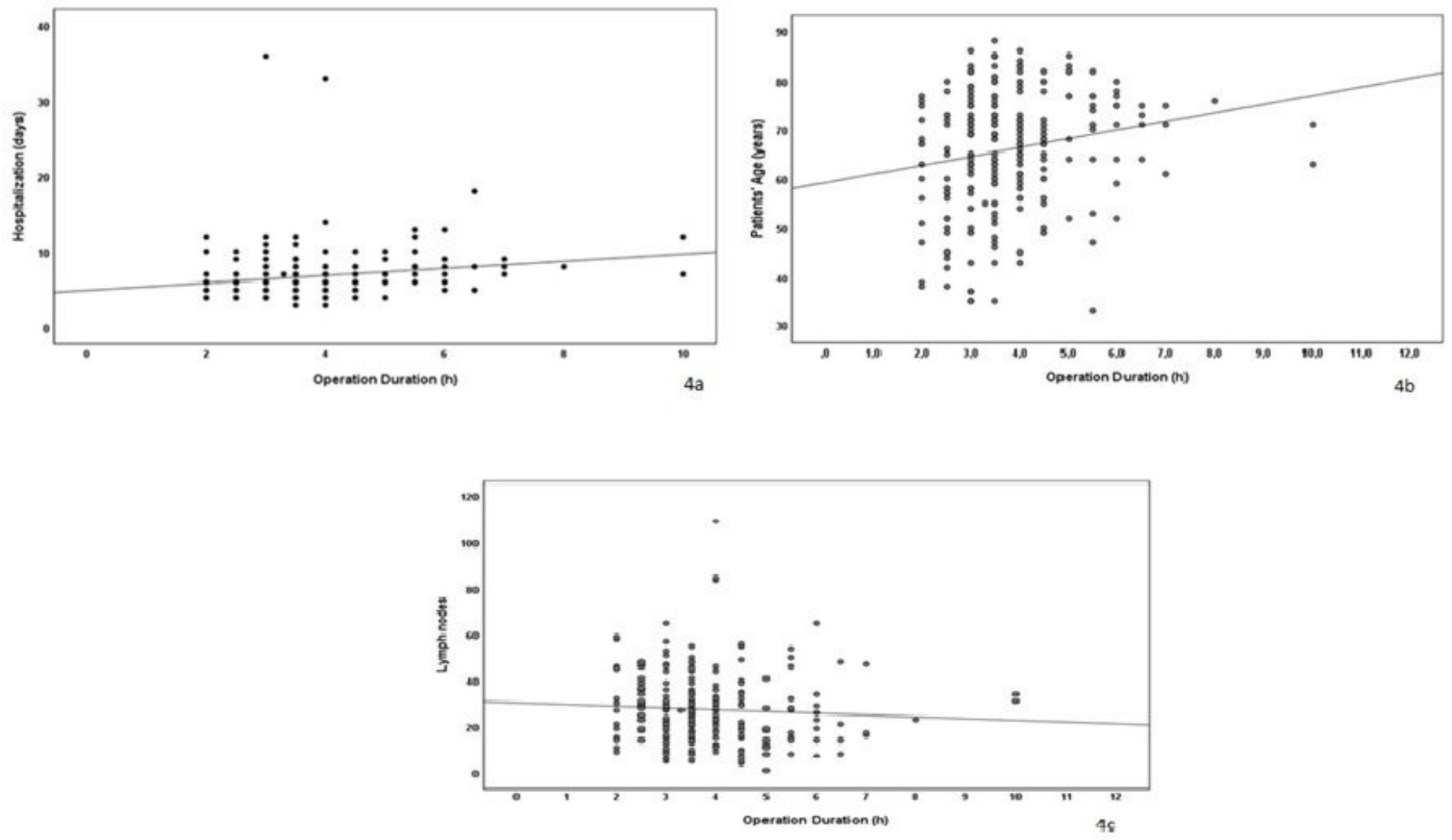

Figure 4

Correlation of operative duration with specific parameters

Correlation of operative duration with a) number of hospitalization days, b) patient's age and c) number of harvested lymph nodes 


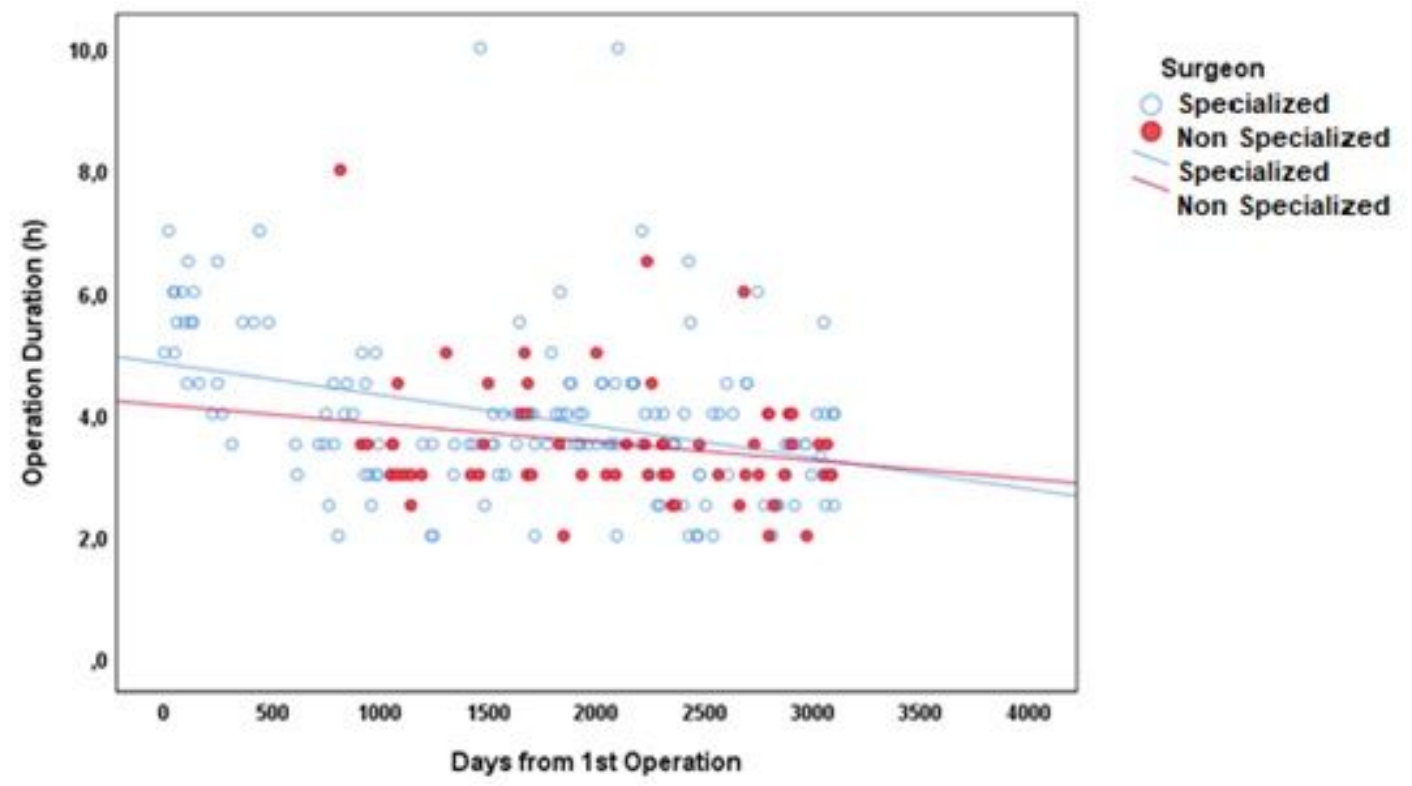

Figure 5

Comparison of the learning effect between specialized and non-specialized surgeons

The learning effect expressed as time (in days) of the operation date minus the $1^{\text {st }}$ recorded operation date

\section{Supplementary Files}

This is a list of supplementary files associated with this preprint. Click to download.

- Dataset.xlsx 